\title{
Photometric comparison of sources of light of different tints
}

\section{A. Crova}

To cite this article: A. Crova (1881) Photometric comparison of sources of light of different tints, Philosophical Magazine Series 5, 12:77, 445-447, DOI: 10.1080/14786448108627133

To link to this article: http://dx.doi.org/10.1080/14786448108627133

曲 Published online: 28 Apr 2009.

Submit your article to this journal ๘

Џll Article views: 2

Q View related articles $\sqsubset$ 
were afterwards slowly modified; and ten hours after the formation of the flower some small black spots were distinguished upon the metals. The observation had to bo discontinued on account of the lateness of the hour; and the next moruing several of the petals had burst.

I ought to mention that the glycerine liquid which was used for this experiment was of very indifferent quality, perhaps because the oleate employed was not prepared with the requisite care, or because Price's glycerine is nowalays not so pure as it was formerly. But this nay have been an advantage; for with an excellent liquid the modifications of the flower would have been too slow.

If after withdrawing the flower from the liquid, and while all the petals are still colourless, they be successively burst in the middle by a wire heated in the flame of a spirit-lamp, a numerons series of brilliant little liquid masses are instantly seen attached to the entire metallic contour, which threads them like the beads of a necklace: their arrangement is very nearly regular; and there are fourteen of them to each petal.

I seize this occasion to insist on two precautions to be taken in making the oleate of soda, precautions indispensable if we wish to form good glycerine liquid. In the first place, the oleic acid obtained at one stage of the preparation must be most carefully protected from the action of the oxygen of the air; in the second place, the sea-salt employed to separate the oleate at the end of the operations must have been perfectly purified. Let us add that, when the oleate is prepared, we must take care not to dissolve it in alcohol to render it more pure; I believe I have proved that, on the contrary, this spoils it.

Let us, in conclusion, with respect to the experiment of the flower, mention a curious instance of persistence of impressions, an instance moreover which is not unprecedented*. Мy wife, whose eyes are very sharp, had observed the flower at different times during the day. Now, at her waking on the morrow, casting her eyes upon the white curtains of the bed, she silw the image of the flower: one of the petals was so clearly delineated that she distinguished even the wire forming its outline; the iuterior was striped with white and yellow; the other petals were more or less indistinct. Changing the direction of her gaze caused the image to disappear.- Extrait des Bulletins te l'Academie Royale de Belgique, sér. 3 , t. ii. no. 7 (1881).

\section{PHOTOMETRIC COMPARISON OF SOURCES OF LIGHT OF DIFEERENT' TINT'S. BY A. CROVA.}

The exact measurement of the ratio of the intensities of two lights of which the emission-temperatures are very different presents great difficulties, on account of the difference of composition of the two lights which are to be compared. The equality of intensity of either the shadows or the illuminated regions of the photometric screen cannot be exactly determined, because of the differences

* See Stevelley, L'Institut, 1859, no. 1309, p. 38; Gorini, Ann. di Ottalm. t. iii. p. 164.

Phil. Mag. S. 5. Vol. 12. No. 77. Dec. 1881. 2L 
of their tints. The employment of a spectrophotometer permits this difficulty to be surmounted.

Let us suppose the simple radiations which constitute the light emanating from an electric regulator and that from a standard Carcel lamp spread out in two contiguous spectra. If the distances of the two sources from the photometer be such that their mean illumination is the same, the two spectra will be far from presenting the same aspect: that of the carbon points is more intense towards the violet extremity, and less luminous towards the red, than that of the lamp. The temperature of emission of the electric light being much higher than that of the lamp, the ratio of the intensities of the simple radiations of the electric light to the intensities of the corresponding lights of the spectrum of the flame of the lamp, will with equality of mean illumination, be represented by a fraction greater than unity towards the violet; but if the red be approached, the ratio will gradually diminish, following the law of continuity, and will be less than unity at the red end.

There exists, then, a determinate simple radiation (whose wavelength depends on the nature of the lights compared) for which that ratio is exactly equal to unity. If this radiation be precisely known, the measurement of the ratio of its intensities in the two spectra will give excatly the ratio of the total intensities of the two sources.

I have constructed a very simple apparatus which permits the practical realization of the theoretic conception of which I have just spoken. Let us illuminate the half-disks of the screen of a Foncault's photometer with an electric light and that of a standard Carcel lamp : the two illuminated regions being placed tangentially, it is very difficult to judge of the equality of the illumination, one of the moieties having a bluish tint in comparison with the other, which appears to be of an orange-yellow; and the standard lamp can be displaced within pretty wide limits without the eye being able to judge if equality of illumination is really obtained.

Let us look at the screen, placing in front of the eye two Nicol prisms the cross sections of which are rectangular, and between which is placed, perpendicular to the axis, a quartz plate of 9 millim. thickness. The eye then sees the two fields coloured of a green tint washed with white; and if we vary the distance" of the standard lamp, a moment arrives when, equality of intensity being obtained, the line of demarcation of the two fields disappears. A very slight variation of the distance of the lamp then suffices to give rise to a very sharp contrast between the two regions.

The thickness of the quartz plate has been calculated so that its interposition between the two rectangular nicols give rise, in the spectra of the two sources, to two large interference-bands situated in the two extremities of the spectrum. On passing from these two bands towards the middle of the spectrum, the intensity of the different preserved radiations varies as the square of the cosine of the angle made by the cross section of the second nicol with the planes of polarization of the different radiations, which have undergone rotatory dispersion in the quartz plate. There is therefore one of them for which the square of the cosine is equal to unity, and which suffers no weakening. It is conceived that it is pos- 
sible empirically to displace the second nicol (and thereby the two bands) so as to preserve the maximum of intensity to the simple radiation the comparison of which would give the same result as that of the total intensity. The neighbouring radiations are more and more rapidly weakened in proportion as their distance from this maximum increases; and this circumstance diminishes the influence of their differences of intensity (which increase with their distance from the maximum) to such a degree as to render then negligible.

In practice the system of nicols and quartz plate is fixed in the body of a small telescope placed opposite to the screen of the photometer, upon the cone through the apex of which we view it. This instrument, constructed with much precision by M. Duboscq, tried with the Drummond light, an electric regulator or sun-light, comparatively with the standard Carcel, immediately gave very precise results in spite of the difference of tint of those different lights.-Comptes Rendus de l'Académie des Sciences, Sept. 26, 1881, t. xciii. pp. 512, 513.

\section{A NEW DEMONSTRATION OF RIEMANN'S THEOREM.}

BY M. CROULLEBOIS.

M. Clausius* has presented Riemann's theorem as a consequence of Green's formula; that important proposition can be demoustrated more directly. The theorem is thus enunciated by Clausius :-

Given two conducting bodies A and $\mathrm{B}$, which may either be insulated or connected with the earth by a conductor; in the vicinity of these let there be any number whatever of other conducting bodies put into communication with the earth by means of conductors. While $\mathrm{B}$ is in communication with the earth and A is insulated, this latter body is charged with electricity up to a certain potential-level $\mathrm{K}$. Let $\mathrm{Q}_{b}^{a}$ be the quantity of electricity accumulated by influence upon $\mathrm{B}$ under these circumstances. A is afterwards put into communication with the earth, while B is insulated and charged with electricity to the same potential-level $\mathrm{K}$. Let $\mathrm{Q}_{a}^{b}$ be the quantity of electricity accumulated upon A by influence under these circumstances. We shall have

$$
Q_{a}^{b}=Q_{b}^{a} \text {. }
$$

Let the two conductors $A$ and $\mathbf{B}$ be initially accompanied by any number of conductors, all connected with the earth - that is to say, at the potential zero. Let us insulate $A$ and bring it to the potential $V_{1}$; the effect of this increase of potential will be to raise the charge of $A$ from 0 to $Q_{a}^{a}$, and to induce charges upon the other conductors, and in particular upon $\mathrm{B}$ the charge $\mathrm{Q}_{b}^{a}$; we can put

$$
\mathrm{Q}_{a}^{a}=\mathrm{C}_{a}^{a} \mathrm{~V}_{1} \text {, and } \mathrm{Q}_{b}^{a}=\mathrm{C}_{b}^{u} \mathrm{~V}_{1} \text {, }
$$

$\mathrm{C}_{a}^{a}$ being the capacity of $\mathrm{A}$, and $\mathrm{C}_{b}^{a}$ the coefficient of induction of $A$ upon B.

Let us discharge $A$ by putting it into communication with the earth, and bring it to the potential $V_{2}$; there comes in like manner: in this second state of equilibrium, for the charges acquired upon $B$ and induced upon $A$ :-

$$
\mathrm{Q}_{b}^{b}=\mathrm{C}_{b}^{b} \mathrm{~V}_{2}, \quad \mathrm{Q}_{a}^{b}=\mathrm{C}_{a}^{b} \mathrm{~V}_{2},
$$

* Theorie Mécanique de la Chaleur, t. ii. pp. 38-44. 